\title{
TET2 Mutations in Ph-Negative-Myeloproliferative Neoplasms: Identification of Three Novel Mutations and Relationship with Clinical and Laboratory Findings
}

\author{
Andrea Patriarca ${ }^{1}$, Donatella Colaizzo ${ }^{2}$, Gianluca Tiscia ${ }^{2}$, Raffaele Spadano ${ }^{1}$, Silvia Di Zacomo ${ }^{3}$, \\ Antonio Spadano ${ }^{3}$, Ida Villanova ${ }^{3}$, Maurizio Margaglione ${ }^{4}$, Elvira Grandone ${ }^{2}$, Alfredo Dragani ${ }^{1}$ \\ ${ }^{1}$ Bleeding and Thrombotic Disorders Unit, Department of Hematology, Ospedale Civile Dello “Spirito Santo”, Pescara, Italy; \\ ${ }^{2}$ Atherosclerosis and Thrombosis Unit, IRCCS Casa Sollievo Della Sofferenza, San Giovanni Rotondo, Italy; ${ }^{3}$ Laboratory of Mo- \\ lecular Biology, Department of Hematology, Ospedale Civile Dello “Spirito Santo”, Pescara, Italy; ${ }^{4}$ Department of Medical Genetics, \\ Foggia University, Foggia, Italy. \\ Email: andreapatriarca80@gmail.com
}

Received December 21 ${ }^{\text {st }}, 2012$; revised February $1^{\text {st }}, 2013$; accepted February $10^{\text {th }}, 2013$

Copyright (C) 2013 Andrea Patriarca et al. This is an open access article distributed under the Creative Commons Attribution License, which permits unrestricted use, distribution, and reproduction in any medium, provided the original work is properly cited.

\begin{abstract}
High-throughput DNA sequence analysis was used to screen for TET2 mutations in peripheral blood derived DNA from 97 patients with BCR-ABL-negative-myeloproliferative neoplasms (MPNs). Overall six mutations in the coding region of the gene were identified in 7 patients with an overall mutational frequency of $7.2 \%$. In polycythemia vera patients (n = 25) were identified 2 mutations (8\%); in those with essential thrombocythemia $(\mathrm{n}=55) 2$ mutations (3.6\%); in those with unclassifiable MPN $(n=8) 3$ mutations (37.5\%). No primary myelofibrosis patiens $(n=6)$ harboured TET2 mutations. Three unreported mutations were identified (p.P177fs, p.C1298del, p.P411del) the first two in patients with unclassifiable MPN, the last in a patient with essential thrombocythemia. On multivariate analysis the diagnosis of an unclassifiable MPN was significantly related to the presence of TET2 mutations ( $\mathrm{p}=0.02$; OR: 2.81; 95\% CI 1.11 - 7.06). We conclude that TET2 mutations occur in both JAK2V617F-positive and -negative MPN and are more frequent in MPN-U patients. This could represent the biological link between the different classes of myeloid malignancies.
\end{abstract}

Keywords: MPN; Methylation; TET2; Molecular Biology

\section{Introduction}

This Philadelphia negative-myeloproliferative neoplasms (MPN) are a spectrum of clonal disorders of the hematopoietic system characterized by overproduction of mature blood elements, a trend to thrombotic and/or hemorrhagic complications with variable rates of transformation to secondary myelofibrosis and acute leukemia [1]. The presence of JAK2 or MPL mutations represents major diagnostic criteria in the WHO classification of classic BCR-abl negative MPNs. However, a variable percentage of patients lack both molecular markers. The molecular basis of JAK2- and MPL-negative MPN remains largely unexplained. Recently, new molecular markers have been described in a vast array of myeloid cancers [1-3]. In particular, alterations in the TET2 gene, a putative tumor suppressor gene located at chromosomal region $4 \mathrm{q} 24$, have been identified in $7 \%-13 \%$ of MPN patients, in 19\% - 26\% myelodysplastic syndromes (MDS), in $12 \%$ - $24 \%$ of acute myeloid leukemia (AML), in $20 \%$ - 40\% of chronic myelomonocytic leukemia (CMML), and in $29 \%$ of systemic mastocytosis [2-9]. Moreover, at the best of our knowledge, no significant correlation was observed between the TET2 mutation status and both the clinical-laboratory phenotype and the risk of secondary clonal evolution in MPNs [6]. Aims of our study were: 1) to investigate the presence of TET2 mutations in MPN patients with or without the JAK2 V617F mutation; 2) to establish a possible relationship between clinical and laboratory findings in the context of Polycythemia Vera (PV), Essential Thrombocytemia (ET), Primary Myelophibrosis (PMF) and Myeloproliferative Neoplasms unclassifiable (MPN-U).

\section{Materials and Methods}

\subsection{Patients}

After approval by the institutional review board, we se- 
lected from our database 98 MPNs adult patients (26 PV; 55 ET; 6 prefibrotic and 3 fibrotic PMF and $8 \mathrm{MPN}-\mathrm{U}$ ), diagnosed according to WHO 2008 diagnostic Criteria [10]. At the time of enrolment, 8 patients showed clinical and laboratory pictures compatible with a secondary evolution as follow: 3 post-ET PMF, 2 post-PV PMF, 1 $\mathrm{PV}$ in accelerated phase, $1 \mathrm{PMF}$ evolved in CMMoL and $1 \mathrm{MPN}-\mathrm{U}$ in secondary fibrosis. All parameters used for statistical analysis, except for those addressing prognosis (survival, leukemic or fibrotic transformation), were those obtained at the time of diagnosis and before any therapeutic intervention.

\subsection{Molecular Biology}

Mutation screening for JAK2 V617F was performed on granulocyte DNA from peripheral blood samples (PD) at Laboratory of our Department, according to the procedure previously described [11]. High-throughput DNA sequence analysis was used to screen for TET2 mutations in PB-derived DNA at Atherosclerosis and Thrombosis Unit, IRCCS Casa Sollievo della Sofferenza, S. Giovanni Rotondo. Briefly, all the exons, introns and 5'UTR of the gene were amplified using forward and reverse PCR primer designed according to the DNA sequence reported in the literature (reference sequence NC 000004). PCR primers were designed to amplify and sequence $<500$ bp amplicons, and overlapping PCR amplicons were designed for all exons $>400$ bp to ensure complete coverage. For each PCR reaction, $50 \mathrm{ng}$ of genomic DNA was used. All the PCR product was sequenced on ABI PRISM 3100 Genetic Analyzer sequencer (PE Biosystems, Foster City, CA, USA). All the frameshift and non sense mutation were scored as pathological mutation. Point mutations were excluded if they were synonymous mutation or included in SNP database

(http://www.ncbi.nlm.gov/project/SNP). DNA was available from 97 patients for TET2 sequencing. In one case (suffering with PV complicated by portal vein thrombosis), we were unable to extract DNA due to sample unsuitability.

\subsection{Statistical Analyses}

Statistical analyses were performed using MedCalc 11.6.1 (1993-2011, Mariackerke, Belgium, Europe). All $\mathrm{p}$ values were two tailored and the level of significance was set at the level of $\mathrm{p}<0.05$. Continuous variables were shown as median and range. Categorical variables were described as frequency and percentage. Comparison between categorical variables was performed by Chisquared statistics. Comparison between categorical and continuous variables was performed by Mann-Whitney U-test or Kruskal-Wallis test, where appropriate. The association between the variables selected from univari- ate analysis and the TET2 mutational status was explored using logistic regression, using as confounding factors age, gender, JAK2 V617F mutation frequency, monocyte, Plt and WBC count, Hb, cellularity on bone marrow trephine biopsy and cytoreductive treatment.

\section{Results}

The diagnostic and clinical characteristics of the whole cohort sorted by diagnosis are summarized in Table 1.

In addition to the expected differences in full blood count, frequency of splenomegaly, JAK2 V617F allele burden, bone marrow cellularity, depending on specific MPN subtype, we found that the PV patients were significantly older than the other patients $(\mathrm{p}=0.04)$ and ET patients were more often females $(\mathrm{p}=0.04)$.

Overall, we found 6 mutations all in the coding sequence of the gene in 7 patients (Table 2) with an overall prevalence of $7.2 \%$. No intron boundaries mutations were found. No significant difference was observed between patients with/without TET2 mutations as far as the JAK2V617F frequency is concerned (Table 3). The same conclusion was reached when we correlated the JAK2V617F allele burden with TET2 mutation status both in the whole cohort and after the exclusion of PV patients.

Interestingly, 3 patients harboured novel TET2 mutations: 2 in the exon 3 (P39 and P98) and 1 in the exon 7 (P42) mutations. The first (p.P177fs) is a frameshift mutation caused by the deletion of c-DNA cytosine 530, the second (p.P411del) is a proline deletion due to the c.12321234delCCT. The third novel mutation (p.C1298del) in the exon 7 is due to the deletion of c.3890_3892delGAT.

The other three mutations identified are reported in Table 2 and were previously described. A significantly higher prevalence of TET2 mutations was recorded in the MPN-U (3/8, 37.5\%) with a OR of 12.72 (95\% CI: 2.21 73.2; $\mathrm{p}=0.005)$. A Plt count below the whole group median count $\left(615 \times 10^{6} / \mathrm{l}\right)$ and a minor frequency of spontaneous MK colony growth were observed in patients carrying TET2 mutations ( $\mathrm{p}=0.02$ (OR: 8.58; 95\% CI 1.1 - 74.4) and $p=0.01$ (OR: 10.3; 95\% CI: 1.19 - 89.87), respectively). On multivariate analysis, the presence of TET2 mutation was significantly and independently associated only with MPN-U diagnosis ( $\mathrm{p}=0.02$ (OR: 2.81; 95\% CI 1.11 - 7.06)).

Twenty-nine major thrombotic events were recorded at diagnosis in the whole cohort (Table 1): 3 of them (10.3\%) occurred in patients carrying TET2 mutation. The first event was an acute myocardial infarction recorded in a 43 years old patient diagnosed with ET; the other 2 patients were both affected by PV and showed a deep vein thrombosis at diagnosis. As far as the 25 thrombotic events in patients without TET2, 3 portal vein 
Novel Mutations and Relationship with Clinical and Laboratory Findings

Table 1. Clinical and laboratory characteristic of $98 \mathrm{MPN}$ at diagnosis according to diagnosis.

\begin{tabular}{|c|c|c|c|c|c|}
\hline & IMF & MPN-U & PV & TE & $p$ value \\
\hline Number of patients & 9 & 8 & 26 & 55 & na \\
\hline Median age, years (range) & $68(54-82)$ & $69(43-67)$ & $72(30-85)$ & $63(25-89)$ & 0.04 \\
\hline Follow-up days, median (range) & $885.5(397-2130)$ & $1314(350-2707)$ & $1358.5(128-5871)$ & $2137(179-4708)$ & 0.1 \\
\hline$H b, \mathrm{~g} / 100 \mathrm{ml}$ (range) & $15.1(12.1-17.4)$ & $16.1(12.5-19.1)$ & $18.0(14.5-21.5)$ & $14.9(11.1-18.1)$ & 0.0001 \\
\hline$W B C \times 10^{9} /$ (range $)$ & $7.43(5.52-13.82)$ & $7.73(5.4-15.53)$ & $10.98(1.7-19.6)$ & $8.3(3.18-16.71)$ & 0.05 \\
\hline Monocytes $\times 10^{9} /$ ( range) & $0.48(0.08-0.89)$ & $0.4(0.3-0.82)$ & $0.45(0.02-1.55)$ & $0.45(0.11-0.78)$ & 0.8 \\
\hline Plt $\times 10^{9} /$ l (range $)$ & 615 (429 - 1094) & $629(181-1006)$ & $481(134-1745)$ & $684(443-1750)$ & 0.0001 \\
\hline JAK2 V617F burden median \% (range) & $40(5-64)$ & $17(4-40)$ & $60(24-90)$ & $14(5-59)$ & 0.0001 \\
\hline EEC $n(\%)$ & $6 / 9(66.6)$ & $6 / 8(75)$ & 23/26 (88.5) & $41 / 55(74.5)$ & 0.1 \\
\hline$C G U-M K n(\%)$ & 3/9 (33.3) & $4 / 8(37.5)$ & $16 / 26(61.5)$ & $36 / 55(65.5)$ & 0.1 \\
\hline$C D 34+S P \times 10^{9} /$ (range $)$ & $0.051(0.013-0.25)$ & $0.021(0.0046-0.051)$ & $0.040(0.0035-0.5)$ & $0.024(0.0015-0.61)$ & 0.07 \\
\hline CD34 + SP (\%) (range) & $0.05(0.035-0.45)$ & $0.03(0.01-0.09)$ & $0.04(0.01-0.56)$ & $0.03(0.0015-0.27)$ & 0.07 \\
\hline Bone marrow cellularity \% (range) & $60(30-90)$ & $40(20-80)$ & $80(60-95)$ & $50(20-98)$ & 0.0001 \\
\hline Complex karyotype $(n)$ & $0 / 9$ & $0 / 8$ & $0 / 26$ & $1 / 54$ & \\
\hline Trisomy $(n)$ & $0 / 9$ & $0 / 8$ & $1 / 26$ & $0 / 54$ & 0.3 \\
\hline Palpable splenomegaly ( $n$ ) & $6 / 9$ & $3 / 8$ & $16 / 26$ & $15 / 55$ & \\
\hline Arterial thromboses (n) & $5 / 9$ & $0 / 8$ & $6 / 26$ & $12 / 55$ & 0.04 \\
\hline Venous thromboses $(n)$ & $1 / 9$ & $0 / 8$ & $6 / 26$ & $3 / 55$ & 0.6 \\
\hline Spontaneus haemorrhage (n) & $0 / 9$ & $0 / 8$ & $1 / 26$ & $1 / 55$ & 07 \\
\hline Post thraumatic haemorrhage (n) & $1 / 9$ & $0 / 9$ & $0 / 26$ & $1 / 55$ & \\
\hline Cytoreductive treatment (n) & $7 / 9$ & $7 / 8$ & $26 / 26$ & $42 / 55$ & 0.06 \\
\hline
\end{tabular}

Table 2. TET2 mutations in MPN patients.

\begin{tabular}{|c|c|c|c|c|}
\hline & Sequence location & Effect & cDNA & Patient ID (diagnosis) \\
\hline p.P177fs & Exon 3 & Frameshift & c.530delC & P39 (MPN-U) \\
\hline p.C1298del & Exon 7 & Deletion & c.3890_3892delGAT & P42 (MPN-U) \\
\hline p.Q652fs ${ }^{*}$ & Exon 3 & Frameshift & c.1954delC & P65 (MPN-U) \\
\hline p.R1572W $W^{*}$ & Exon 11 & Missense & c. $4714 \mathrm{C}>\mathrm{T}$ & P23 (PV) \\
\hline p.V1718L ${ }^{*}$ & Exson 11 & Missense & c. $5152 \mathrm{G}>\mathrm{T}$ & P21, P25 (TE, PV) \\
\hline
\end{tabular}

*Novel mutations.

thrombosis, 1 Budd-Chiari syndrome, 4 Strokes, 2 deep vein thromboses and 13 acute myocardial infarctions were recorded. As stated before in 1 case of myocardial infarction information about TET2 mutation was not 
Table 3. Clinical and laboratory features of 97 MPNs patients stratified by TET2 status.

\begin{tabular}{|c|c|c|c|}
\hline & TET2 positive MPN (n = 7) & TET2 negative MPN $(n=90)$ & p value \\
\hline \multicolumn{4}{|l|}{ Diagnosis } \\
\hline$M P N-U(n=8)$ & $3(37.5 \%)$ & $5 / 8(62.5 \%)$ & \\
\hline$E T(n=55)$ & $2(3.6 \%)$ & $53(96.4 \%)$ & 0.005 \\
\hline$P V(n=25)$ & $2(8.0 \%)$ & $23(92 \%)$ & \\
\hline $\operatorname{IMF}(n=9)$ & 0 & 9 & \\
\hline Median age, years (range) & $71(43-76)$ & $65.5(25-89)$ & 0.57 \\
\hline Follow-up months, median (range) & $38.6(13.3-130)$ & $54.2(4.3-195.7)$ & 0.38 \\
\hline$H b, \mathrm{~g} / 100 \mathrm{ml}$ (range) & $16.1(13.3-19.9)$ & $15.2(11.1-21.5)$ & 0.22 \\
\hline$W B C \times 10^{9} / 1$ (range) & $6.86(5.4-12.4)$ & $8.56(1.7-19.6)$ & 0.3 \\
\hline Monocytes $\times 10^{9} / 1$ (range) & $0.3(0.1-1.15)$ & $0.45(0.02-0.9)$ & 0.45 \\
\hline Plt $\times 10^{9} /$ l (range) & $476(134-849)$ & $629(149-1750)$ & 0.02 \\
\hline JAK 2 V617F n (\%) & 6/7 (85.7) & 66/90 (73.3) & 0.78 \\
\hline JAK 2 V617F burden median \% (range) & $24(5-65)$ & $25(5-90)$ & 0.99 \\
\hline EEC $n(\%)$ & $4 / 7(57.1)$ & $71 / 90$ (78.8) & 0.39 \\
\hline$C F U-M K n(\%)$ & 1/7 (14.2) & $57 / 90(63.3)$ & 0.01 \\
\hline$C D 34+S P \times 10^{9} / 1$ (range $)$ & $0.021(0.0103-0.273)$ & $0.03(0.0015-0.61)$ & 0.89 \\
\hline CD34 + SP (\%) (range) & $0.03(0.02-0.35)$ & $0.035(0.0015-0.56)$ & 0.43 \\
\hline Bone marrow cellularity \% (range) & $50(30-80)$ & $50(20$ - 98) & 0.96 \\
\hline Single cytogenetic abnormality ( $n$ ) & $0 / 7$ & $5 / 90(5.5)$ & \\
\hline Complex karyotype (n) & $0 / 7$ & $1 / 90(1.1)$ & 0.49 \\
\hline Trisomy $(n)$ & $0 / 7$ & $1 / 90(1.1)$ & \\
\hline Pruritus (n) & $2 / 7$ & $21 / 90$ & 0.88 \\
\hline Palpable splenomegaly ( $n$ ) & $2 / 7$ & $37 / 90$ & 0.8 \\
\hline Splenic square $\mathrm{cm}^{2}$ (range) & $40(31-105)$ & $42(25-200)$ & 0.45 \\
\hline Arterial thromboses ( $n$ ) & $1 / 7$ & $21 / 90$ & 0.43 \\
\hline Venous thromboses ( $n$ ) & $2 / 7$ & $7 / 90$ & \\
\hline Spontaneus haemorrhage (n) & $0 / 7$ & $2 / 90$ & 0.58 \\
\hline Post thraumatic haemorrhage (n) & $0 / 7$ & $2 / 90$ & \\
\hline Cytoreductive treatment $(n)$ & 7/7 & $86 / 90$ & 0.66 \\
\hline
\end{tabular}

available.

Four bleeding episodes (2 post traumatic and 2 spontaneous) were recorded during follow-up, all in patients without TET2 mutation. The post traumatic haemorrhages were as follows: 1 episode post-hysterectomy in IMF patient, 1 in ET patient following caesarean section due to dystocic labour. Both patients had received lowmolecular weight-heparin as standard prophylaxis for deep vein thrombosis at the time of haemorrhagic complication. Moreover, only in ET patient a Plt count approaching $1.000 \times 10^{9} / \mathrm{l}$ was recorded.

Two patients showed a spontaneous haemorrhage: the first was a PV patient with subdural haemorrhage, the latter was an ET patient. In both cases a Plt count above $1.000 \times 10^{9} / 1$ was recorded without significant other coagulation abnormality. 
Eight patients (8.2\%) showed clinical-laboratory findings compatible with secondary evolution (3 post-ET MF, 2 post-PV MF one of which underwent allogenic stem cell transplantation, 1 post-PV accelerated phase, 1 evolution into CMMoL, $1 \mathrm{MPN}-\mathrm{U}$ evolving to secondary fibrosis) after a median follow-up of 68.2 months (range 32.4 - 146.2 months). In this subgroup, only one PV patient (P21) evolving into accelerated phase carried one of the TET2 mutations. Moreover, in the whole cohort we registered only one death due to post-surgical sepsis (in 1 patient suffering from PV).

\section{Discussion}

The TET2 mutational frequency observed in this study $(7.2 \%)$ is similar to that reported by other authors $[3,6,12]$ in both in sporadic and in familiar MPNs. Moreover, our data confirm data by Tefferi et al. [6] about the lower frequency of TET2 mutations in patients without JAK2 V617F.

Interestingly, we find three novel mutations in the coding sequence of TET2, two located within the exon 3 and one on exon 7. The first mutation is a deletion of a cytosine 530 leading to a frameshift and possibility to a premature stop codon resulting in a non-functional protein. The second one is a tri-base deletion from 1232 to 1234 resulting in the p.P411del. The last one is a GAT deletion from 3890 to 3892 on c-DNA. The exact effect of these last two deletions is unknown but they likely result in an abnormal protein folding. The other TET2 mutations previously reported by other authors, with the exception of p.Q652fs, have never been reported in MPNs patients [12-20]. p.Q652fs was previously described $[12,13]$ in a familiar ET with double heterozygous TET2 status evolving after a brief observation into secondary AML and in a type I CMML). p.R1572W was for the first time identified by Kohlmann et al. in a type I CMML [14]; p.V1718L was described by several groups [15-20] mainly in MDS and MPN/MDS.

In agreement with Tefferi et al. [8], we find a significant correlation between TET2 mutation and an MPN-U diagnosis. Even if the small sample size does not allow firm conclusion, we can argue that TET2 is the biological substrate linking MPNs, MPN/MDS, MDS and AML. This hypothesis is supported by the absence of a significant difference in clinical and laboratory features between TET2 mutated and w-t patients in MPNs and by the increasing frequency of the mutation passing from trough the different classes of diseases. No other significant differences were found between clinical and laboratory data at diagnosis sampling the cohort by TET2 mutation and diagnosis. More in detail, our data do not confirm the correlation with the age in MPNs, underlined by Tefferi et al. [6]. This is probably due to the lower preva- lence (8\%) of the mutation in PV group, which in our sample is older than the other groups.

Moreover, according to data previously published by Nguyen-Khac in transformed MPNs, the presence of any TET2 mutation does not relate to other genetic aberration detected with karyotype and FISH examination on chromosome from bone marrow hematopoietic precursors [13].

Due to the low number of patients progressing to secondary fibrosis or secondary hematopoietic malignancy, we were not able to evaluate OS and EFS and thus if TET2 mutations are a detrimental or a favourable factor in secondary clonal evolution in MPNs.

Our data confirm and extend those previously published and allow us to speculate that TET2 mutations could represent the biological link between different classes of myeloid malignancies.

\section{REFERENCES}

[1] A. Tefferi and W. Vainchenker, "Myeloproliferative Neoplasms: Molecular Pathophysiology, Essential Clinical Understanding, and Treatment Strategies,” JCO, Vol. 29, No. 5, 2011, pp. 573-582. http://dx.doi.org/10.1200/JCO.2010.29.8711

[2] O. Abdel-Wahab, A. Mullally, C. Hedvat, G. Garcia-Manero, J. W. M. Patel, M. Wadleigh, et al., "Genetic Characterization of TET1, TET2, and TET3 Alterations in Myeloid Malignancies,” Blood, Vol. 114, No. 1, 2009, pp. 144-147.

http://dx.doi.org/10.1182/blood-2009-03-210039

[3] F. Delhommeau, S. Dupont, V. Della Valle, C. James, S. Trannoy, A. Massé, et al., "Mutation in TET2 in Myeloid Cancers," The New England Journal of Medicine, Vol. 360, 2009, pp. 2289-2301.

http://dx.doi.org/10.1056/NEJMoa0810069

[4] L. Couronné, E. Lippert, J. Andrieux, O. Kosmider, I. Radford-Weiss, D. Penther, et al., "Analyses of TET2 Mutations in Post-Myeloproliferative Neoplasm Acute Myeloid Leukemias,” Leukemia, Vol. 24, No. 1, 2009, pp. 201-203.

[5] S. M. Langemeijer, R. P. Kuiper, M. Berends, R. Knops, M. G. Aslanyan, M. Massop, et al., "Acquired Mutations in TET2 Are Common in Myelodysplastic Syndromes," Nature Genetics, Vol. 41, 2009, pp. 838-842. http://dx.doi.org/10.1038/ng.391

[6] A. Tefferi, A. Pardanani, K. H. Lim, O. Abdel-Wahab, T. L. Lasho, J. Patel, et al., "TET2 Mutations and Their Clinical Correlates in Polycythemia Vera, Essential Thrombocythemia and Myelofibrosis," Leukemia, Vol. 23, 2009, pp. 905-911. http://dx.doi.org/10.1038/leu.2009.47

[7] A. Tefferi, R. L. Levine, K. H. Lim, O. Abdel-Wahab, T. L. Lasho, J. Patel, et al., "Frequent TET2 Mutations in Systemic Mastocytosis: Clinical, KITD816V and FIP1L1PDGFRA Correlates,” Leukemia, Vol. 23, 2009, pp. 900904. http://dx.doi.org/10.1038/leu.2009.37 
[8] A. Tefferi, K. H. Lim, O. Abdel-Wahab, T. L. Lasho, J. Patel, M. M. Patnaik, et al., "Detection of Mutant TET2 in Myeloid Malignancies Other Than Myeloproliferative Neoplasms: CMML, MDS, MDS/MPN and AML,” Leukemia, Vol. 23, 2009, pp. 1343-1345. http://dx.doi.org/10.1038/leu.2009.59

[9] A. Wahab, "Genetics of the Myeloproliferative Neoplasms," Current Opinion in Hematology, Vol. 18, 2011, pp. 117-123. http://dx.doi.org/10.1097/MOH.0b013e328343998e

[10] A. Tefferi and J. M. Vardiman, "Classification and Diagnosis of Myeloproliferative Neoplasms: The 2008 World Health Organization Criteria and Point-of-Care Diagnostic Algorithms,” Leukemia, Vol. 22, 2008, pp. 14-22. http://dx.doi.org/10.1038/sj.leu.2404955

[11] A. Patriarca, F. Pompetti, R. Malizia, O. Iuliani, I. Di Marzio, A. Spadano, et al., "Is the Absence of JAK2 Mutation a Risk Factor for Bleeding in Essential Thrombocythemia? An Analysis of 106 Patients,” Bloodtransfusion, Vol. 8, 2010, pp. 21-27.

[12] F. Nguyen-Khac, C. Lesty, V. Eclache, L. Couronnè, O. Kosmider, J. Andrieux, et al., "Chromosomal Abnormalities in Transformed Ph-Negative Myeloproliferative Neoplasms are Associated to the Transformation Subtype and Independent of JAK2 and the TET2 Mutations," Genes, Chromosomes \& Cancer, Vol. 49, No. 10, 2010, pp. 919927. http://dx.doi.org/10.1002/gcc.20802

[13] C. Saint-Martin, G. Leroy, F. Delhommeau, G. Panelatti, S. Dupont, C. James, et al., "Analysis of the Ten-Eleven Translocation 2 (TET2) Gene in Familial Myeloproliferative Neoplasms,” Blood, Vol. 114, No. 8, 2009, pp. 1628-1632. http://dx.doi.org/10.1182/blood-2009-01-197525

[14] O. Kosmider, V. Gelsi-Boyer, M. Ciudad, C. Racoeur, V. Jooste, N. Vey, et al., "TET2 Gene Mutation Is a Frequent and Adverse Event in Chronic Myelomonocytic Leukemia,” Haematologica, Vol. 94, No. 12, 2009, pp. 1676-1681.

http://dx.doi.org/10.3324/haematol.2009.011205

[15] A. Kohlmann, H. U. Klein, S. Weissmann, S. Bresolin, T.
Chaplin, H. Cuppens, et al., “The Interlaboratory Robustness of Next-Generation Sequencing (IRON) Study: A Deep Sequencing Investigation of TET2, CBL and KRAS Mutations by an International Consortium Involving 10 Laboratories,” Leukemia, Vol. 25, 2011, pp. 1840-1848. http://dx.doi.org/10.1038/leu.2011.155

[16] M. T. Voso, E. Fabiani, A. Piciocchi, C. Matteucci, L. Brandimarte, C. Finelli, et al., "Role of BCL2L10 Methylation and TET2 Mutations in Higher Risk Myelodysplastic Syndromes Treated with 5-Azacytidine,” Leukemia, Vol. 25, 2011, pp. 1910-1913. http://dx.doi.org/10.1038/leu.2011.170

[17] M. Ko, Y. Huang, A. M. Jankowska, U. J. Pape, M. Tahiliani, H. S. Bandukwala, et al., "Impaired Hydroxylation of 5-Methylcytosine in Myeloid Cancers with Mutant TET2,” Nature, Vol. 468, 2010, pp. 839-843. http://dx.doi.org/10.1038/nature09586

[18] D. A. Pollyea, A. Raval, B. Kusler, J. R. Gotlib, A. A. Alizadeh and B. S. Mitchell, "Impact of TET2 Mutations on mRNA Expression and Clinical Outcomes in MDS Patients Treated with DNA Methyltransferase Inhibitors," Hematological Oncology, Vol. 29, No. 3, 2011, pp. 157160. http://dx.doi.org/10.1002/hon.976

[19] H. Szpurka, A. M. Jankowska, H. Makishima, J. Bodo, N. Bejanyan, E. D. His, et al., "Spectrum of Mutations in RARS-T Patients Includes TET2 and ASXL1 Mutations," Leukemia Research, Vol. 34, No. 8, 2010, pp. 969-973. http://dx.doi.org/10.1016/j.leukres.2010.02.033

[20] A. M. Jankowska, H. Szpurka, R. V. Tiu, H. Makishima, M. Afable, J. Huh, et al., "Loss of Heterozygosity 4q24 and TET2 Mutations Associated with Myelodysplastic/ Myeloproliferative Neoplasms,” Blood, Vol. 113, No. 25, 2009, pp. 6403-6410. http://dx.doi.org/10.1182/blood-2009-02-205690

[21] D. Olcaydu, E. Rumi, A. Harutyunyan, F. Passamonti, D. Pietra, C. Pascutto, et al., "The Role of the JAK2 GGCC Haplotype and the TET2 Gene In Familial Myeloproliferative Neoplasms,” Haematologica, Vol. 96, No. 3, 2011, pp. 367-374.

http://dx.doi.org/10.3324/haematol.2010.034488 